\title{
Confiabilidade estrutural de uma ponte protendida de madeira considerando o tráfego real
}

\author{
Structural reliability of prestressed timber bridges \\ considering real traffic
}

\section{Andrés Batista Cheung \\ Ricardo de Mello Scaliante \\ Malton Lindquist \\ André Luis Christoforo \\ Carlito Calil Junior}

\section{Resumo \\ A}

$\mathrm{s}$ pontes protendidas de madeira consistem em sistemas estruturais alternativos, mais leves do que as pontes tradicionais, e apresentam grande rigidez e facilidade de montagem. No Brasil a primeira ponte protendida de madeira foi projetada e construída sobre o Rio

Monjolinho, em São Carlos, SP. A confiabilidade desse tipo de estrutura é um dos pontos de maior preocupação técnica, embora essa forma de avaliação ainda não seja utilizada em projetos de pontes de madeira. Este trabalho objetivou avaliar a confiabilidade estrutural do sistema laminado protendido de madeira da ponte projetada sobre o Rio Monjolinho, com foco especial na resistência à flexão e na perda de protensão do sistema, considerando-se para tanto um conjunto variado de trens-tipo. $\mathrm{O}$ tabuleiro foi avaliado como uma viga equivalente, baseado no comportamento de placa ortotrópica, e para verificar a segurança da ponte um estudo de confiabilidade foi realizado considerando ações reais obtidas por meio dos dados da concessionária Centrovias. Os resultados das análises de

Andrés Batista Cheung Universidade Federal de Mato Grosso do Sul

Campo Grande - MS - Brasil

Ricardo de Mello Scaliante Departamento Nacional de Infraestrutura de Transportes de Mato Graosso do Sul

Campo Grande - MS - Brasil Malton Lindquist
Secretaria da Fazenda do Estado do Fortaleza - CE - Brasil

André Luis Christoforo Universidade Federal de São Carlos São Carlos - SP - Brasil

Carlito Calil Junior Universidade de São Paulo São Carlos - SP - Brasil

Recebido em 16/06/15 Aceito em 15/09/16 confiabilidade revelaram que a ponte protendida de madeira apresentou índices de confiabilidade compatíveis para a maioria dos carregamentos simulados do tráfego, embora para alguns tipos de caminhões a ponte tenha apresentado índices de confiabilidade abaixo do recomendado pelas normas internacionais.

Palavras-chaves: Pontes de madeira. Protensão. Confiabilidade.

\section{Abstract}

Prestressed wooden bridges are alternative structural systems that are lighter than traditional bridges and present high stiffness and portability. In Brazil, the first prestressed timber bridge was designed and built over the Monjolinho River (São Carlos - SP). The reliability of this structure is a major technical concern, even though this kind of assessment is not yet used in timber bridge projects. The aim of this research study was to evaluate the structural reliability of the laminated prestressed system of the timber bridge designed for the Monjolinho River, with special focus on the system's bending strength and prestess losses, considering a varied set of live loads. The deck was assessed as an equivalent beam, based on orthotropic plate behaviour. To check the safety of the bridge, a reliability study was conducted considering actual actions obtained from the data provided by Centrovias, the road's concession company. The results of the reliability tests showed that the prestressed timber bridge presented reliability rates compatible with most simulated traffic loads, but for some types of trucks the bridge presented reliability indices below those recommended by international standards.

Keywords: Timber bridges. Prestressing. Reliability.

CHEUNG, A. B.; SCALIANTE, R. de M.; LINDQUIST, M.; CHRISTOFORO, A. L.; CALIL JUNIOR, C. Confiabilidade estrutural 221 de uma ponte protendida de madeira considerando o tráfego real. Ambiente Construído, Porto Alegre, v. 17, n. 2, p. 221-232, abr./jun. 2017.

ISSN 1678-8621 Associação Nacional de Tecnologia do Ambiente Construído.

http://dx.doi.org/10.1590/s1678-86212017000200154 


\section{Introdução}

No Brasil pequenas pontes de estradas vicinais são essenciais para o transporte de produtos agrícolas, e uma boa alternativa consiste no uso de sistemas em pontes protendidas de madeira, que são mais leves do que as pontes tradicionais e apresentam grande rigidez e facilidade de montagem. Esse tipo de ponte foi originalmente desenvolvido em Ontário, no Canadá, na década de 1970, como forma alternativa de reabilitação de tabuleiros laminados pregados de pontes antigas (CHEUNG; LINDQUIST; CALIL JUNIOR, 2004). No Brasil a primeira ponte de madeira laminada protendida projetada foi construída sobre o Rio Monjolinho e está localizada na região da cidade São Carlos, interior do estado de São Paulo (FONTE, 2004).

A madeira laminada protendida consiste de uma série de peças de madeira serrada dispostas lado a lado e comprimidas transversalmente por barras de protensão de alta resistência (GÓES et al., 2006; CALIL JUNIOR, 2006). A força de compressão transversal aplicada pelas barras de protensão atua solidarizando as lâminas, como ilustra a Figura 1. Como vantagem do sistema pode-se citar a facilidade de pré-fabricação, o peso próprio reduzido, quando comparado a outros materiais, e o comportamento de placa.

A solidarização das lâminas de madeira pela protensão confere à placa formada um comportamento anisotrópico, e nesse sentido diversos pesquisadores demonstram que os tabuleiros protendidos podem ser tratados como placas ortotrópicas (RITTER, 1990; TAYLOR; BATCHELOR; VAN DALEN, 1982; OLIVA; DIMAKIS, 1988; OKIMOTO, 1997; CHEUNG; CALIL JUNIOR, 2006). Alguns métodos utilizam a transformação do problema de placa ortrotópica para uma viga com largura efetiva, referente à distribuição de carregamento. Para a aplicação dessa metodologia faz-se necessária a obtenção dos parâmetros elásticos equivalentes, tais como os módulos de elasticidade longitudinais em duas direções perpendiculares $(E x, E y)$ e o módulo de torção no plano longitudinal-transversal (Gxy). Nesse modelo a complexidade do tabuleiro da ponte é reduzida para uma largura efetiva. Devido à simplicidade e ao grau razoável de aproximação, esse modelo ainda é apresentado pelas normas nacionais e internacionais como um possível modelo de cálculo. Muitos são os fatores que influenciam a determinação da largura efetiva: nível de protensão, propriedades ortrotópicas da madeira, deformação lenta da madeira, variação de umidade e tipo de sistema de protensão (CHEUNG; CALIL JUNIOR, 2006).

Existem na literatura vários tipos pontes protendidas de madeira (GÓES; DIAS, 2005). No Brasil a grande maioria foi projetada sem o recurso de análises baseadas em confiabilidade, visto que a norma brasileira NBR 7190 (ABNT, 1997) não apresenta as premissas, os métodos de cálculo e os índices de confiabilidade para a análise de estruturas considerando-se o critério de confiabilidade estrutural (CHEUNG; PINTO; CALIL JUNIOR, 2012).

Com o intuito de evidenciar a importância do estudo da confiabilidade em estruturas de madeira projetadas ou a ser projetadas, esta pesquisa objetivou avaliar a confiabilidade estrutural da ponte de madeira laminada protendida projetada construída sobre o Rio Monjolinho, em São Carlos, SP, com ênfase na resistência à flexão e na perda de protensão do sistema.

Figura 1 - Arranjo básico de tabuleiros laminados protendidos

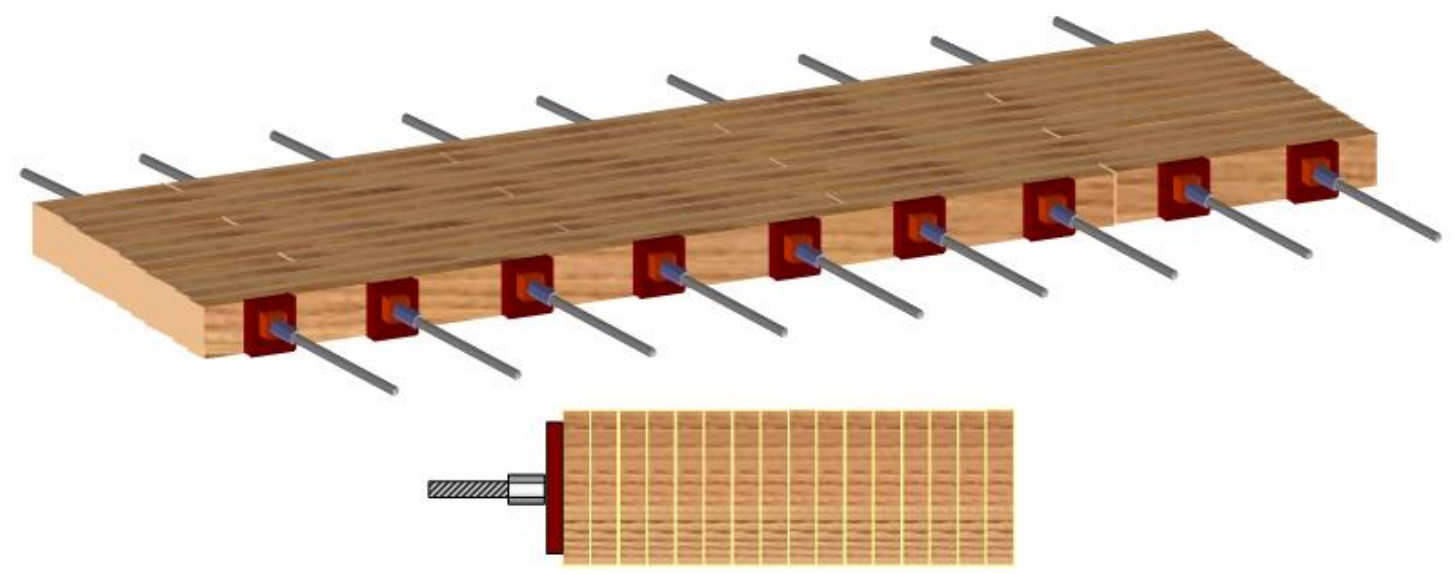

222 Cheung, A. B.; Scaliante, R. de M.; Lindquist, M.; Christoforo, A. L.; Calil Junior, C. 


\section{Material e métodos}

A ponte Monjolinho consiste em uma placa biapoiada com uma via de tráfego de $8 \mathrm{~m}$ de comprimento, $4,5 \mathrm{~m}$ de largura e $25 \mathrm{~cm}$ de espessura. Essa estrutura feita de madeira serrada de reflorestamento (Eucalyptus citriodora) foi construída em 2003 pelo Programa Emergencial das Pontes de Madeira para o Estado de São Paulo, financiado pela Fundação de Amparo à Pesquisa do Estado de São Paulo (Fapesp). As peças foram tratadas com preservante óxido hidrossolúvel CCA (arseniato de cobre cromatado), e o sistema de protensão utilizado foi o Dywidag, com diâmetro das barras de $15 \mathrm{~mm}$ (ST 85/105). Essa ponte foi a primeira estrutura de madeira protendida construída na América do Sul (Figura 2) e possui localização (coordenadas do GPS) S 21 59' 25.5 W 47 53' 30.6 e altitude de 849 m.

Figura 2 - Ponte sobre o Rio Monjolinho (a) e esquema estrutural idealizado (b)

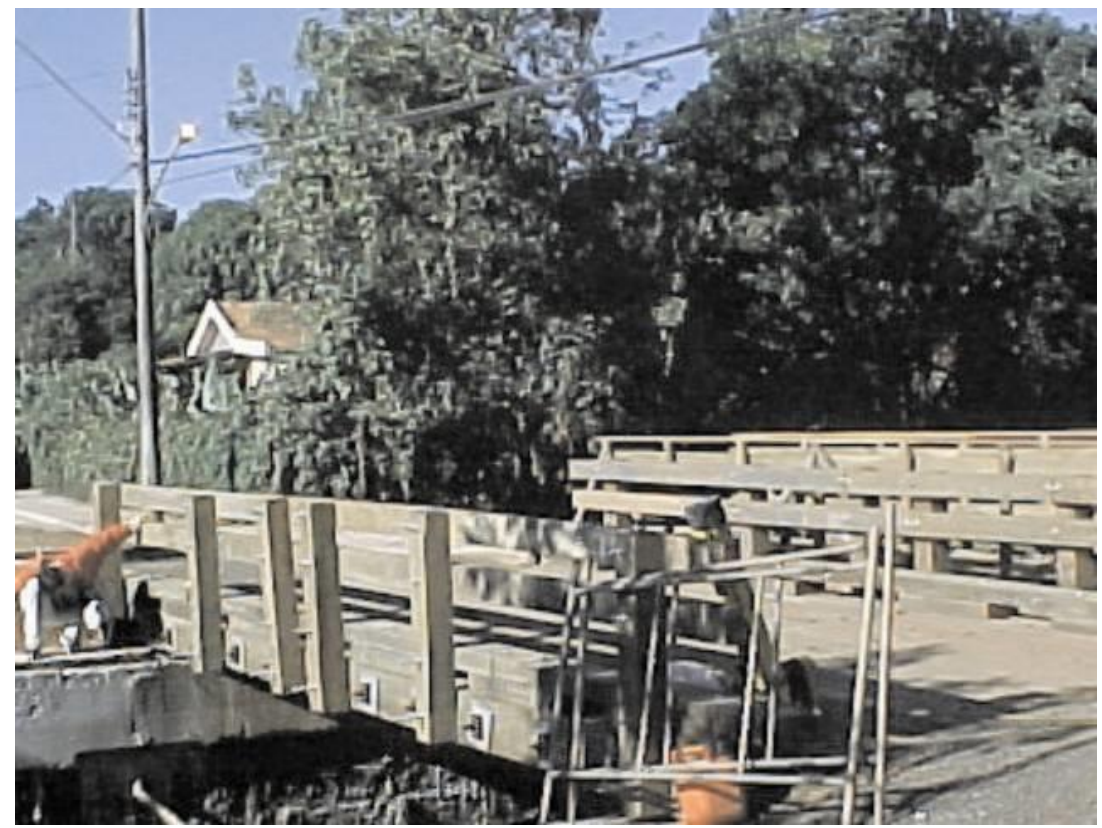

(a) Localização: GPS coordenadas S 21 59' 25.5" e W 47 53' 30.6", altitude de 849 m

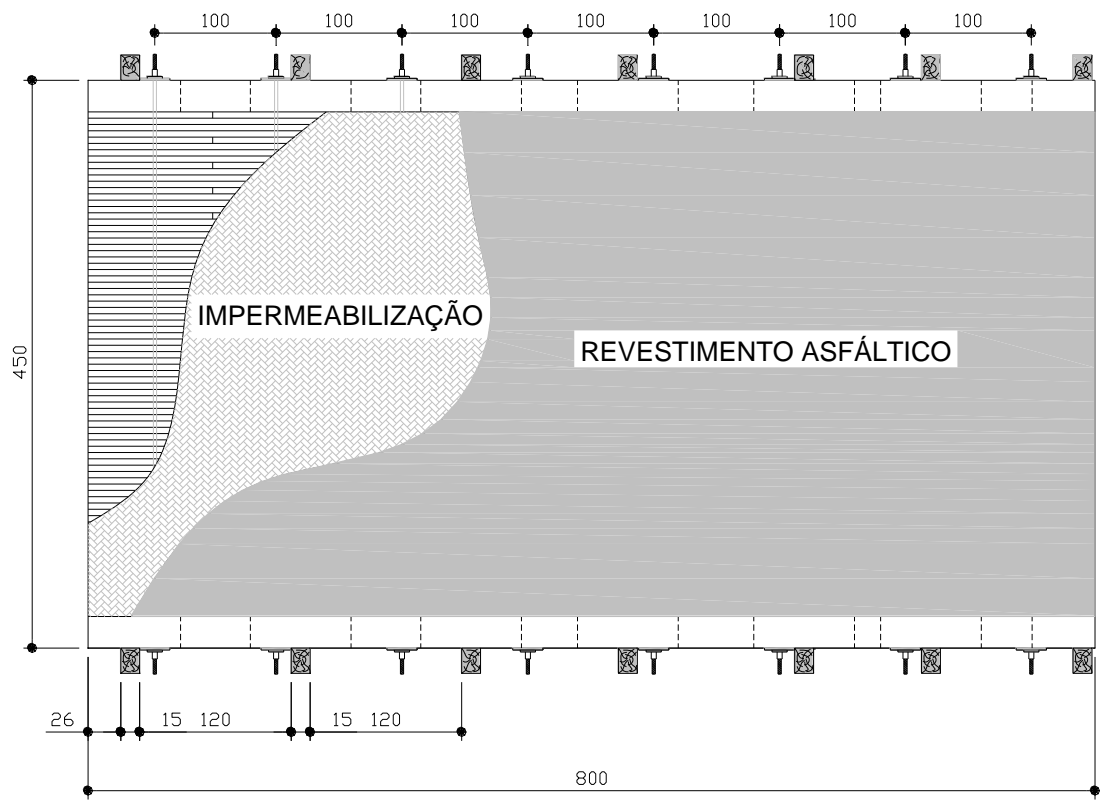

(b)

Fonte: adaptado de Fonte (2004). 


\section{Modelo estrutural e modos de ruptura do sistema}

Dois modos de falha têm sido relatados na literatura (RITTER, 1990). A Figura 3 ilustra os dois principais tipos: flexão transversal, produzindo tendência para a abertura entre as lâminas na parte inferior da placa (Figura 3a); e cisalhamento, produzindo a tendência de as lâminas deslizarem entre si verticalmente (Figura 3b). Os aspectos mais importantes de ruptura do sistema estão relacionados com as resistências das lâminas de madeira, elementos de protensão, ancoragens e perdas de protensão com o tempo. É recomendável manter um nível mínimo de protensão como um dos principais aspectos de construção e manutenção dessas pontes.

Neste trabalho o tabuleiro foi analisado como uma viga, assumindo-se que uma linha da roda do veículo é distribuída por uma largura efetiva, representada por $D_{w}$ (Figura $3 \mathrm{c}$ ). O valor é baseado no comportamento ortotrópico da placa. $\mathrm{O}$ efeito das juntas de topo na distribuição da carga depende da frequência das juntas e do nível de protensão $\left(\sigma_{p i}\right)$, e é expresso por um fator junta de topo $C_{b j}$ (Figura 3d), de acordo com Ritter (1990). Os esforços de flexão longitudinal da placa e os deslocamentos controlam a espessura necessária.

Também é apresentada uma expressão analítica (Equação 4) para a determinação da largura efetiva $D_{w}$ (Figura 3d), baseada na teoria de placas ortotrópicas. Essa expressão foi obtida pela regressão múltipla dos resultados teóricos obtidos analiticamente (Figura 4a) com os parâmetros $\alpha \mathrm{e}$ $\theta$ determinados pelas Equações 1 e 2 respectivamente. Os resultados obtidos pela expressão ajustada são apresentados na Figura 4b. Das Equações 1, 2 e 3, $E_{x}$ é o modulo de elasticidade longitudinal, $E_{y}$ é o modulo de elasticidade transversal, $G_{x y}$ é o módulo de cisalhamento, $b$ é a largura, $t$ é a espessura e $L$ é o vão livre do tabuleiro.

$$
\begin{aligned}
& \alpha=\frac{D_{x y}+D_{y x}+D_{1}+D_{2}}{2 \cdot\left(D_{x} \cdot D_{y}\right)^{1 / 2}}=\frac{D_{x y}+D_{y x}}{2 \cdot \sqrt{D_{x} \cdot D_{y}}}=\frac{D_{x y}}{\sqrt{D_{x} \cdot D_{y}}}=\frac{2 \cdot G_{x y}}{\sqrt{E_{x} \cdot C_{b j} \cdot E_{y}}} \\
& \text { Eq. } 1 \\
& \theta=\frac{b}{2 \cdot L}\left(\frac{D_{x}}{D_{y}}\right)^{1 / 4}=\frac{b}{2 \cdot L}\left[\frac{E_{x} \cdot C_{b j}}{E_{y}}\right]^{1 / 4} \\
& D_{x}=\frac{C_{b j} \cdot E_{x} \cdot t^{3}}{12} ; D_{y}=\frac{E_{y} \cdot t^{3}}{12} ; D_{x y}=D_{y x}=\frac{G_{x y} \cdot t^{3}}{6} \text { e } D_{1}=D_{2}=0
\end{aligned}
$$

Eq. 3

Figura 3 - Falhas típicas: ruptura por flexão transversal (a) e por cisalhamento (b), fator de junta de topo $\left[C_{b j}\right](c)$ e determinação de $D_{w}(\mathrm{~d})$

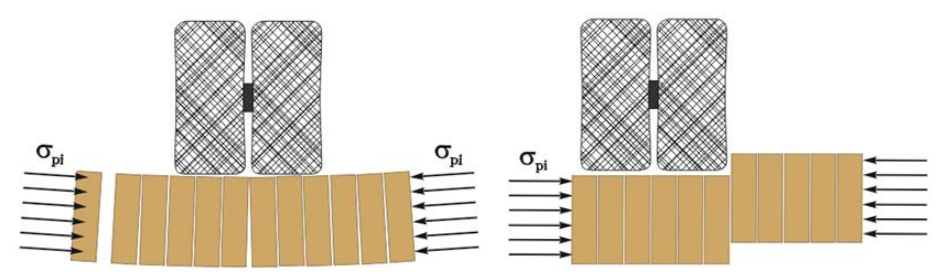

(a)

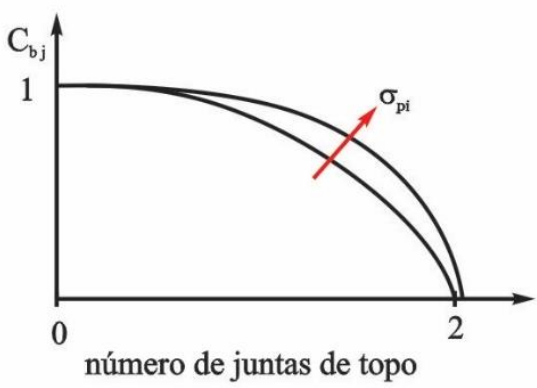

(c)

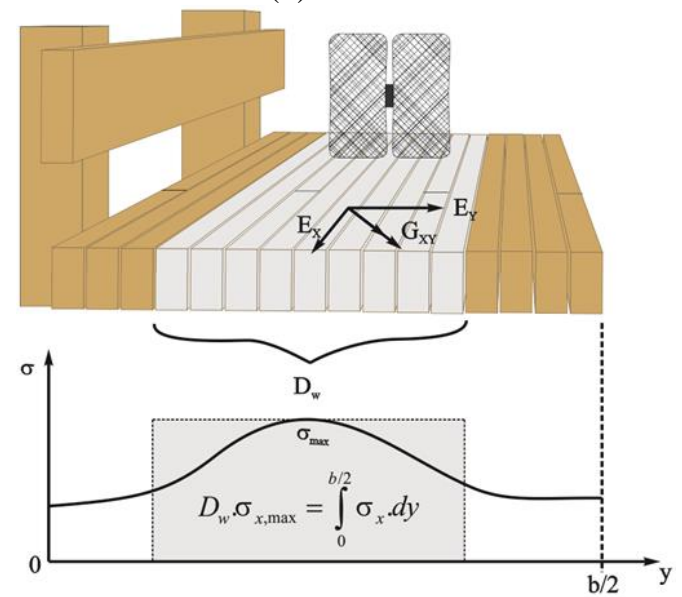

(d)

224 Cheung, A. B.; Scaliante, R. de M.; Lindquist, M.; Christoforo, A. L.; Calil Junior, C. 
Figura 4 - Largura efetiva obtida pela teoria de placa ortotrópica (a) e expressão ajustada (b)

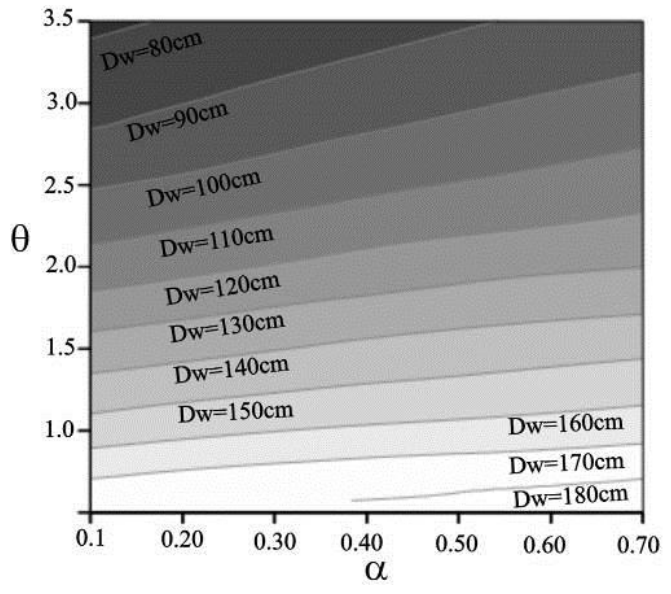

(a)

A Equação 4 mostra que a largura efetiva $\left(D_{w}\right)$ pode ser obtida por meio dos parâmetros $\alpha$ e $\theta$ (Figura 4), onde $D_{w}$ é expresso em centímetros. Embora o valor da largura efetiva necessite apenas dos parâmetros $\theta$ e $\alpha$, outras variáveis são necessárias para a avaliação, tais como o módulo de elasticidade longitudinal, o módulo de elasticidade transversal, o módulo de cisalhamento transversal, o fator de junta de topo e o nível de protensão.

$D_{w}(\alpha, \theta)=27,131 \cdot \alpha-57,295 \cdot \theta+3,463 \cdot \alpha \cdot \theta+5,621$.

$\theta^{2}-13,277 \cdot \alpha^{2}+204,224$

Eq. 4

A comparação entre os resultados numéricos e experimentais realizados por Dahl, Bovim e Malo (2006) com diferentes tipos de metodologias de projeto revelou que a metodologia proposta por Ritter (1990) fornece boa concordância com o comportamento estrutural das placas protendidas de madeira.

A ponte pode ser representada por uma viga simplesmente apoiada, e as tensões normais $\left(\sigma_{x}\right)$ devidas à flexão reta e simples podem ser obtidas por meio da Equação 5, em que $M$ denota o momento fletor, $I_{y}$ é o momento de inércia (eixo de inércia perpendicular ao plano de carregamento) e $z$ é a ordenada de posição medida a partir do eixo neutro da seção transversal. As rodas são posicionadas no meio do vão da viga, com a largura efetiva calculada por meio da Equação 4 , para a avaliação do estado limite último e estado limite de deformação excessiva (RITTER, 1990; DAHL; BOVIM; MALO, 2006; FONTE, 2004).

$\sigma_{x}=\frac{M}{I_{y}} \cdot z$

Eq. 5

O nível de protensão na madeira influencia os parâmetros elásticos, conforme os estudos

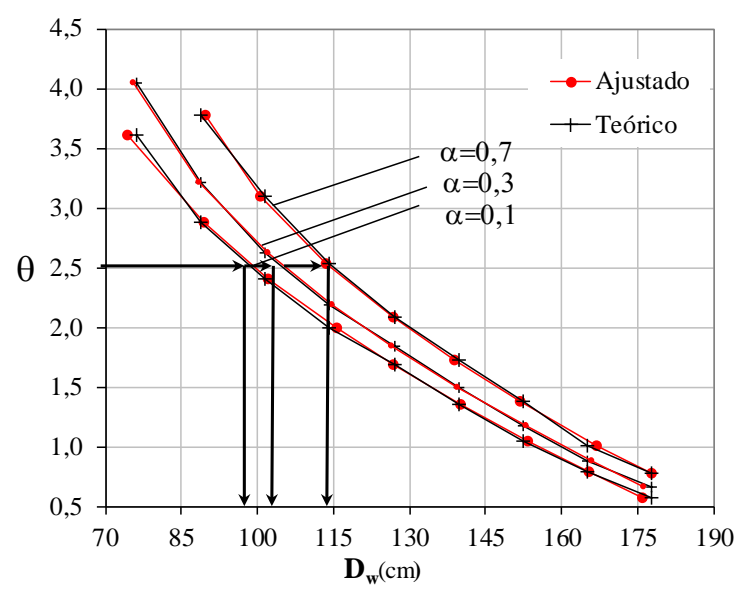

(b)

realizados por Okimoto (1997). Além disso, o autor indica funções que relacionam os parâmetros elásticos com a tensão de protensão, expressos na Equação 6, em que $\phi$ é o fator da perda de protensão, que é uma simplificação do efeito real, e $\sigma_{N}$ é o nível de protensão inicial, expresso em $\mathrm{kN} / \mathrm{m}^{2}$.

$\frac{E_{y}}{E_{x}}=28,78 \cdot 10^{-5} \cdot \varphi \cdot \sigma_{N}-0,001008$

$\frac{G_{x y}}{E_{x}}=3,8 \times 10^{-6} \cdot \varphi \times \sigma_{N}+0,010364$

Eq. 6

As perdas de protensão causadas pela deformação lenta da madeira, o que alivia as tensões nas barras, foram consideradas como variáveis aleatórias. A equação de estado limite último considerada foi definida como o momento devido aos carregamentos móveis e permanentes que excedem a resistência à flexão (MOR), analogamente aos estudos realizados por Eamon $e t$ al. (2000).

Foram analisados três tipos de geometria de eixos (LINDQUIST, 2006), ilustrados na Figura 5. Adotou-se somente o eixo traseiro de um veículo sobre a ponte em razão do seu pequeno comprimento e também por apresentar somente uma única faixa de tráfego.

\section{Modelo de carregamento móvel}

O aumento do limite dos pesos dos caminhões pode acelerar a deterioração do revestimento da ponte e sua segurança estrutural. Assim, neste trabalho o tráfego real foi representado por um modelo de carregamento móvel desenvolvido por meio de registros (realizados e fornecidos pela concessionária Centrovias) no ano de $2000 \mathrm{em}$ estações de pesagem localizadas na rodovia 
Washington Luiz. O estudo forneceu dados estatísticos sobre os pesos brutos dos veículos (GVW - gross vehicle weight) e carga por eixo de uma rodovia de alta capacidade de tráfego, e, apesar de a ponte nesse estudo não ter as mesmas características de tráfego, os dados apresentados neste trabalho representam a distribuição mais provável de carga nos eixos. Isso decorre do fato da inexistência de dados reais para pontes em estradas vicinais. Os caminhões foram separados por geometria dos eixos, como ilustrado na Figura 6.

A partir das frequências obtidas dos dados experimentais foi diagnosticado que os carregamentos seguem distribuições unimodal, bimodal e trimodal. Com isso foram estimadas as distribuições de probabilidade dos caminhões nas condições vazio e totalmente carregado, utilizando-se para isso o método da composição, expresso pela Equação 7, em que $f(x)$ denota a função densidade de probabilidade mista, $p_{i}$ são os pesos $\left(\sum_{i=1}^{m} p_{i}=1\right)$ e $f_{i}(x)[i=1,2, \ldots, m]$ consiste nas funções densidade de probabilidade de variáveis aleatórias.

$$
f(x)=\sum_{i=1}^{m} p_{i} \cdot f_{i}(x)
$$

A Figura 7a ilustra o histograma das cargas do eixo traseiro do caminhão do tipo 3C. Os resultados, analisados com os dados fornecidos pela Centrovias, mostraram que as distribuições unimodal, bimodal e trimodal representaram bem os dados experimentais. Para a obtenção dos pesos e dos parâmetros das distribuições normais foi utilizado o método dos mínimos quadrados (Figura $7 b$ ), que consiste em uma técnica para minimizar os resíduos obtidos das diferenças quadráticas entre os valores observados e estimados.

Figura 5 - Modelo estrutural ajustado para três diferentes tipos de eixo

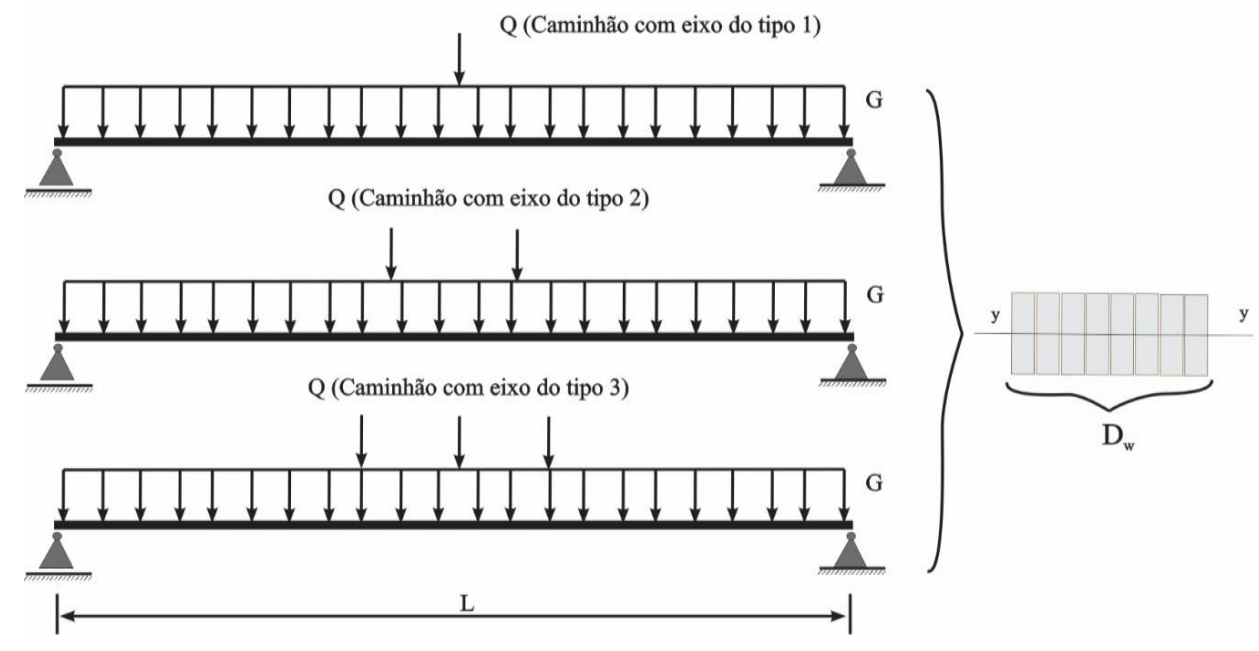

Figura 6 - Modelos de caminhões

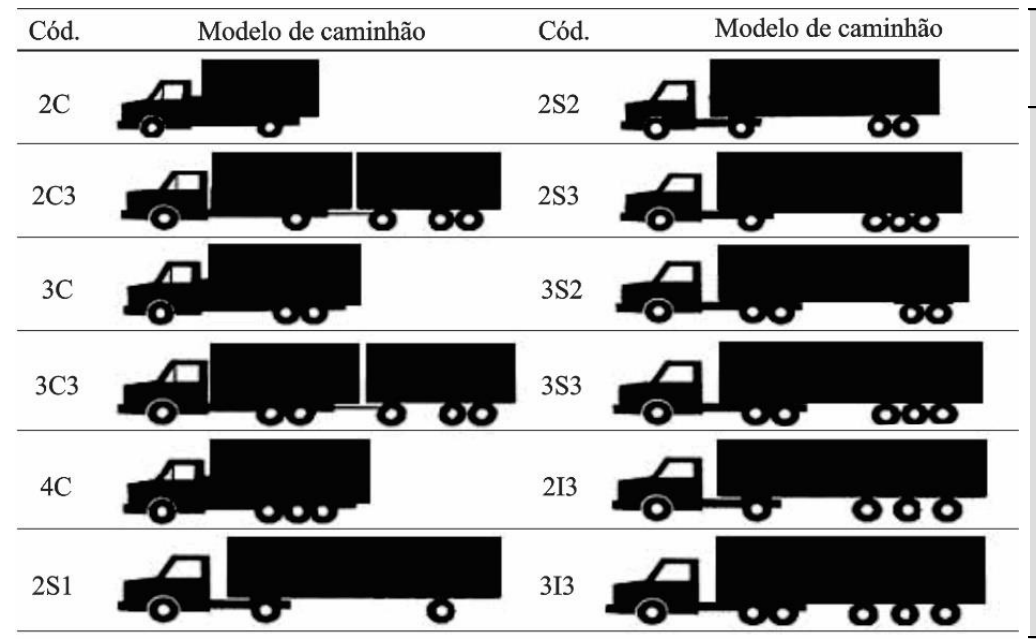

\begin{tabular}{ccc}
\hline código & $\begin{array}{c}\text { Tipo de } \\
\text { eixo }\end{array}$ & $\begin{array}{c}\text { Número de } \\
\text { caminhões }\end{array}$ \\
\hline 2C & $1-1$ & 27950 \\
2C3 & $1-1-1-2$ & 260 \\
3C & $1-2$ & 38910 \\
3C3 & $1-2-1-2$ & 20 \\
4C & $1-3$ & 38 \\
2S1 & $1-1-1$ & 994 \\
2S2 & $1-1-2$ & 4752 \\
2S3 & $1-1-3$ & 36831 \\
3S2 & $1-2-2$ & 318 \\
3S3 & $1-2-3$ & 6030 \\
2I3 & $1-1-1-1-1$ & 2532 \\
3I3 & $1-2-1-1-1$ & 27 \\
& & \\
Nenhum & Indefinido & 1287 \\
\hline
\end{tabular}

Fonte: adaptado de Lindquist (2006).

226 Cheung, A. B.; Scaliante, R. de M.; Lindquist, M.; Christoforo, A. L.; Calil Junior, C. 
Figura 7 - Histograma das cargas do eixo traseiro do caminhão do tipo 3C (a) e função de probabilidade acumulada (b)

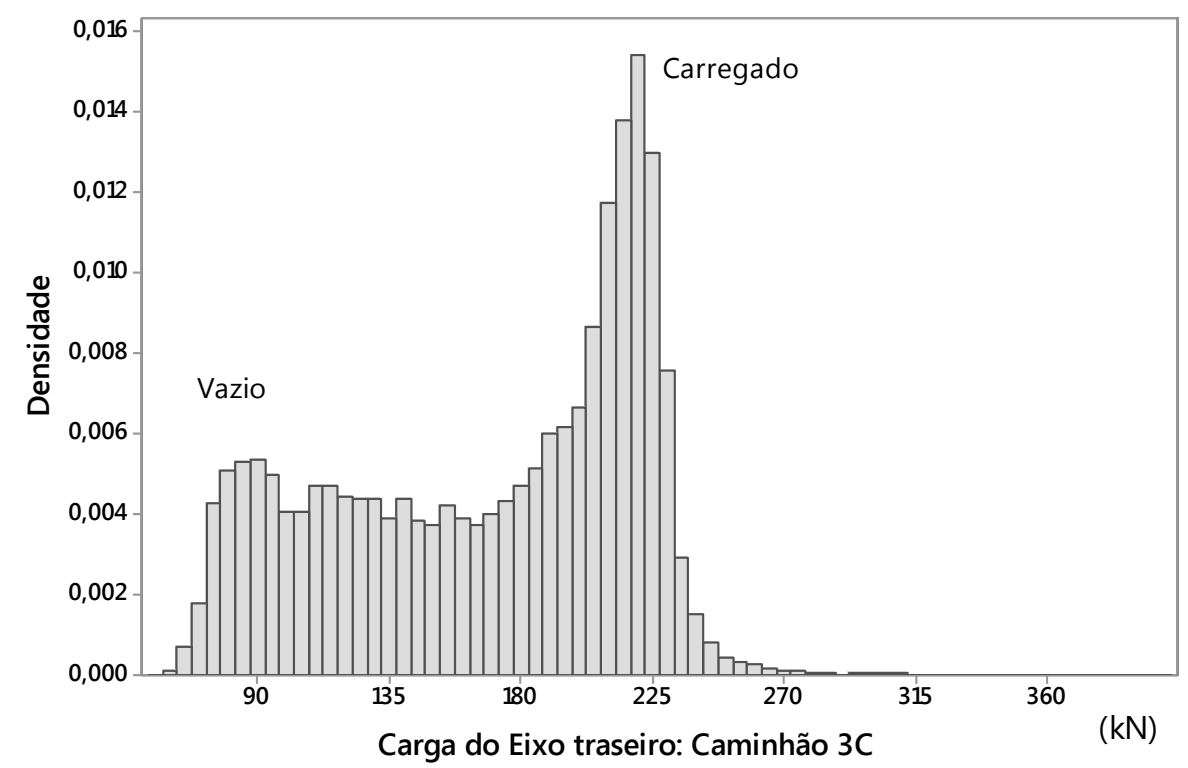

(a)

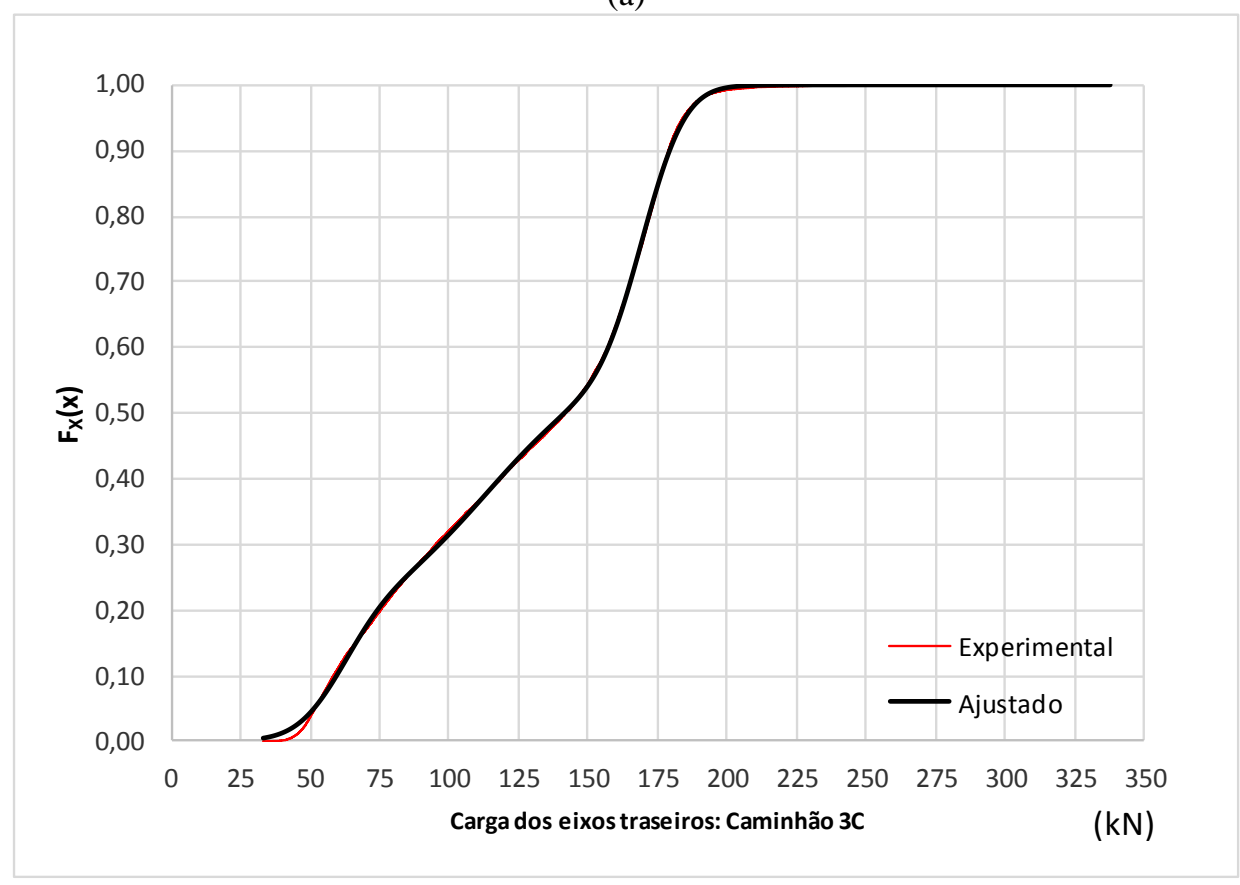

(b)

Fonte: adaptado de Lindquist (2006).

O histograma (que representa o eixo traseiro do caminhão do tipo 3C) aponta para dois tipos mais prováveis de pesos nesse tipo de veículo. O primeiro pico no histograma, com valores ao redor de $50 \mathrm{kN}$, indica provavelmente a carga com o caminhão vazio, sendo o peso nos eixos traseiros de um caminhão do tipo $3 \mathrm{C}$ sem a carroceria de aproximadamente $40 \mathrm{kN}$, de acordo com as especificações encontradas em manuais de fabricantes de veículos dessa natureza.
O segundo pico indica a procura por se utilizar toda a capacidade prevista na legislação, que é de $170 \mathrm{kN}$ para o eixo tandem duplo (LINDQUIST, 2006). A solução encontrada para analisar estatisticamente os dados consistiu em encaixá-los em três distribuições normais, conforme Lindquist et al. (2005). Para uma distribuição que resulta na combinação de três funções normais, a função cumulativa fica expressa por (Eq. 8): 
$\Phi\left(x ; \mu_{1}, \sigma_{1}, \mu_{2}, \sigma_{2}, \mu_{3}, \sigma_{3}\right)=p_{1} \cdot \Phi\left(x ; \mu_{1}, \sigma_{1}\right)+p_{2} \cdot$

$. \Phi\left(x ; \mu_{2}, \sigma_{2}\right)+p_{3} . \Phi\left(x ; \mu_{3}, \sigma_{3}\right) \quad$ Eq. 8

Onde $\Phi(x ; \mu, \sigma)$ é a função de distribuição cumilativa normal, com média $\mu$ e desvio padrão.

A Figura 8 apresenta os parâmetros ajustados com os dados experimentais coletados para cada modelo de caminhão estudado. Os resultados da distribuição de frequências da Figura $7 b$ indicam que a distribuição gaussiana pode ser utilizada para representar os dados experimentais. Dessa forma, os parâmetros das distribuições de probabilidades foram obtidos utilizando o método dos mínimos quadrados para as diferenças entre os valores estimados e os observados.

Como pode ser observado na Figura 8, cada caminhão tem diferentes parâmetros de interação, e em quase todos os veículos o parâmetro principal é para o caminhão completamente carregado. Esse método pode ser usado para estimar os possíveis impactos para pontes de madeira no desenvolvimento de políticas racionais para o transporte agrícola.

Para pontes curtas, como no caso da ponte de madeira em estudo (8 $\mathrm{m}$ de comprimento), os trieixos governam o momento fletor máximo, e dessa forma a diferença entre as configurações torna-se pequena para vão menores que $18 \mathrm{~m}$, como mostrado por Harry et al. (2003). Fica evidente que, em função da pequena dimensão longitudinal da ponte, alguns caminhões não posicionam todos os eixos em um único instante, como no caso do caminhão do tipo $3 \mathrm{~S} 3$, ou podem não ter todos os eixos contribuindo para o momento máximo, como no caso do caminhão do tipo 2S1 (Figura 6).

\section{Equações de estado limite e variáveis aleatórias}

Neste trabalho a equação de estado limite último é definida quando a tensão normal nas fibras inferiores atinge o valor da resistência à flexão da madeira (MOR), e as estatísticas desse parâmetro foram baseadas em ensaios realizados por Fonte (2004). A Equação 9 apresenta a relação de estado limite $(G)$ utilizada neste trabalho. A distância entre eixos foi considerada (de forma simplificada) constante e igual a $122 \mathrm{~cm}$.

$$
G(*)=\theta_{R} \cdot M_{R}(*)-\theta_{E} \cdot M_{E}(*)
$$

Nas Equações de 8 a 10, $\varphi$ é o fator de impacto vertical $(\varphi=1,25$; coeficiente estabelecido pela norma NBR 7190 (ABNT, 1997) para revestimento asfáltico e vão de $8 \mathrm{~m}$ ), $\gamma_{a}$ é o peso específico do asfalto, $\gamma_{w}$ é o peso específico da madeira, $P$ é o peso da metade do eixo, $\mathrm{k}_{\bmod }$ é o coeficiente de modificação total $\left(\mathrm{k}_{\bmod }=0,70\right), t$ é a espessura da placa, $e$ é a espessura do asfalto, $L$ é o vão livre da ponte, e $f_{M}$ é a resistência à flexão obtida por ensaios em peças com dimensões estruturais (FONTE, 2004). Os coeficientes dos modelos de incerteza $\theta_{E}$ (para o efeito das ações) e $\theta_{R}$ (para as resistências) são descritos como variáveis aleatórias com coeficiente de variação de 0,10 . Foi utilizada uma correlação entre $E_{x}$ e $f_{M}$ de 0,70 (LINDQUIST, 2006). A Tabela 1 apresenta as distribuições de probabilidades e os parâmetros utilizados no trabalho. Fonte (2004) construiu e obteve a maioria dos parâmetros utilizados na análise, contudo alguns estudos como perda de protensão, influência dos fatores de junta de topo $\left(C_{b j}\right)$, módulo de ruptura e outros fatores foram obtidos dos trabalhos de Okimoto (1997), Cheung (2003), Lindquist (2006) e JCSS (JOINT..., 2001).

Figura 8 - Parâmetros da distribuição encontrados pelo procedimento de otimização por mínimos quadrados

\begin{tabular}{|c|c|c|c|c|c|c|c|c|c|c|}
\hline Class & Axle & $\mathbf{p}_{1}$ & $\begin{array}{c}\mu_{1} \\
\mathbf{k N} \\
\end{array}$ & $\begin{array}{r}\sigma_{1} \\
\mathbf{k N}\end{array}$ & $\mathbf{p}_{2}$ & $\begin{array}{c}\mu_{2} \\
\text { kN } \\
\end{array}$ & $\begin{array}{r}\sigma_{2} \\
\mathbf{k N} \\
\end{array}$ & $\mathbf{p}_{3}$ & $\begin{array}{r}\mu_{3} \\
\mathbf{k N} \\
\end{array}$ & $\begin{array}{r}\sigma_{3} \\
\mathbf{k N} \\
\end{array}$ \\
\hline \multirow{2}{*}{$2 \mathrm{C}$} & 1 & 0.162 & 17.5 & 2.6 & 0.303 & 26.3 & 4.8 & 0.535 & 32.4 & 9.5 \\
\hline & 2 & 0.080 & 21.0 & 1.7 & 0.435 & 34.0 & 8.7 & 0.485 & 61.9 & 22.0 \\
\hline \multirow{4}{*}{$2 \mathrm{C} 3$} & 1 & 0.064 & 43.6 & 2.8 & 0.936 & 55.8 & 4.2 & & & \\
\hline & 2 & 0.028 & 43.6 & 11.4 & 0.972 & 104.8 & 10.2 & & & \\
\hline & 3 & 0.092 & 38.8 & 9.4 & 0.908 & 91.3 & 12.7 & & & \\
\hline & $4+5$ & 1.000 & 184.5 & 14.6 & & & & & & \\
\hline \multirow{2}{*}{$3 \mathrm{C}$} & 1 & 0.134 & 30.3 & 4.2 & 0.726 & 41.2 & 8.3 & 0.139 & 48.5 & 6.3 \\
\hline & $2+3$ & 0.184 & 61.6 & 12.6 & 0.396 & 114.5 & 33.4 & 0.420 & 169.9 & 11.8 \\
\hline \multirow{4}{*}{$3 \mathrm{C} 3$} & 1 & 1.000 & 51.8 & 2.3 & & & & & & \\
\hline & $2+3$ & 1.000 & 132.3 & 13.3 & & & & & & \\
\hline & 4 & 1.000 & 73.3 & 18.1 & & & & & & \\
\hline & $5+6$ & 1.000 & 180.9 & 27.9 & & & & & & \\
\hline \multirow{2}{*}{$4 C$} & 1 & 1.000 & 75.9 & 24.0 & & & & & & \\
\hline & $2+3+4$ & 1.000 & 234.2 & 61.7 & & & & & & \\
\hline \multirow{3}{*}{$2 S 1$} & 1 & 0.122 & 31.6 & 2.3 & 0.878 & 41.4 & 8.9 & & & \\
\hline & 2 & 0.172 & 40.6 & 2.2 & 0.445 & 48.3 & 9.3 & 0.384 & 66.9 & 20.0 \\
\hline & 3 & 0.304 & 38.0 & 4.5 & 0.696 & 68.6 & 12.7 & & & \\
\hline \multirow{3}{*}{$2 \mathrm{~S} 2$} & 1 & 0.250 & 40.1 & 2.7 & 0.239 & 46.6 & 1.5 & 0.511 & 50.0 & 3.7 \\
\hline & 2 & 0.606 & 50.0 & 8.3 & 0.394 & 90.5 & 14.0 & & & \\
\hline & $3+4$ & 0.433 & 63.1 & 8.3 & 0.540 & 108.1 & 35.8 & 0.027 & 349.5 & 1.7 \\
\hline
\end{tabular}

\begin{tabular}{|c|c|c|c|c|c|c|c|c|c|c|}
\hline Class & Axle & $\mathbf{p}_{1}$ & $\begin{array}{c}\mu_{1} \\
\text { kN }\end{array}$ & $\begin{array}{r}\sigma_{1} \\
\mathbf{k N} \\
\end{array}$ & $\mathbf{p}_{2}$ & $\begin{array}{c}\mu_{2} \\
\text { kN }\end{array}$ & $\begin{array}{r}\sigma_{2} \\
\mathbf{k N} \\
\end{array}$ & $\mathbf{p}_{3}$ & $\begin{array}{c}\mu_{3} \\
\text { kN } \\
\end{array}$ & $\begin{array}{l}\sigma_{3} \\
\mathbf{k N}\end{array}$ \\
\hline \multirow{3}{*}{$2 \mathrm{S3}$} & 1 & 0.071 & 40.6 & 4.2 & 0.811 & 51.6 & 2.2 & 0.118 & 59.1 & 2.2 \\
\hline & 2 & 0.047 & 45.8 & 5.0 & 0.059 & 75.7 & 5.9 & 0.894 & 106.7 & 8.1 \\
\hline & $3+4+5$ & 0.052 & 80.2 & 27.9 & 0.108 & 179.6 & 30.5 & 0.840 & 259.8 & 17.2 \\
\hline \multirow{3}{*}{$3 \mathrm{~S} 2$} & 1 & 0.944 & 50.6 & 5.2 & 0.056 & 53.7 & 3.8 & & & \\
\hline & $2+3$ & 0.522 & 92.6 & 20.7 & 0.478 & 155.7 & 17.5 & & & \\
\hline & $4+5$ & 0.376 & 117.6 & 27.2 & 0.624 & 210.1 & 36.9 & & & \\
\hline \multirow{3}{*}{$3 \mathrm{S3}$} & 1 & 0.878 & 49.6 & 3.8 & 0.122 & 57.2 & 2.2 & & & \\
\hline & $2+3$ & 0.253 & 129.1 & 32.2 & 0.747 & 160.2 & 9.9 & & & \\
\hline & $4+5+6$ & 0.212 & 191.7 & 39.7 & 0.788 & 249.7 & 10.9 & & & \\
\hline \multirow{6}{*}{213} & 1 & 0.254 & 51.8 & 1.6 & 0.208 & 53.7 & 2.3 & 0.538 & 60.0 & 1.6 \\
\hline & 2 & 0.284 & 97.5 & 4.1 & 0.606 & 104.6 & 7.5 & 0.109 & 112.6 & 20.4 \\
\hline & 3 & 0.066 & 65.3 & 7.0 & 0.220 & 83.8 & 5.1 & 0.714 & 99.5 & 4.6 \\
\hline & 4 & 0.045 & 61.2 & 2.7 & 0.955 & 100.6 & 4.3 & & & \\
\hline & 5 & 0.042 & 87.2 & 3.0 & 0.430 & 99.6 & 2.3 & 0.528 & 105.3 & 7.9 \\
\hline & $3+4+5$ & 0.051 & 213.8 & 8.1 & 0.535 & 283.9 & 7.1 & 0.414 & 204.8 & 9.1 \\
\hline \multirow{6}{*}{313} & 1 & 1.000 & 47.1 & 6.6 & & & & & & \\
\hline & $2+3$ & 1.000 & 132.6 & 33.5 & & & & & & \\
\hline & 4 & 1.000 & 83.3 & 25.8 & & & & & & \\
\hline & 5 & 1.000 & 100.8 & 17.3 & & & & & & \\
\hline & 6 & 1.000 & 95.7 & 20.7 & & & & & & \\
\hline & $4+5+6$ & 1.000 & 279.860 & 37.3 & & & & & & \\
\hline
\end{tabular}

228 Cheung, A. B.; Scaliante, R. de M.; Lindquist, M.; Christoforo, A. L.; Calil Junior, C. 
Tabela 1 - Variáveis aleatórias e determinísticas para a análise de confiabilidade

\begin{tabular}{c|c|c|c|c|c}
\hline Variável & Unidade & Dist. de prob. & $\boldsymbol{\mu}_{\mathbf{x}}$ & $\boldsymbol{\sigma}_{\mathbf{x}}$ & Referência \\
\hline$E_{x}$ & $\mathrm{kN} / \mathrm{m}^{2}$ & Log-normal & 1709,00 & 596,53 & Fonte (2004) \\
$\sigma_{N}$ & $\mathrm{kN} / \mathrm{m}^{2}$ & Normal & 0,06 & 0,01 & Fonte (2004) \\
$\phi$ & - & Normal & 0,67 & 0,06 & Fonte (2004) e Cheung (2003) \\
$C_{b j}$ & - & Normal & 0,84 & 0,04 & Okimoto (1997) \\
$t$ & $\mathrm{~m}$ & Uniforme & 0,2475 & 0,0036 & Fonte (2004) \\
$\gamma_{w}$ & $\mathrm{kN} / \mathrm{m}^{3}$ & Normal & 9,63 & 0,69 & Fonte (2004) \\
$\gamma_{a}$ & $\mathrm{kN} / \mathrm{m}^{3}$ & Normal & 24,01 & 2,25 & Ritter (1990) \\
$P$ & $\mathrm{kN}$ & Normal & \multicolumn{2}{|c|}{ Figura 7} & Lindquist (2006) \\
$f_{M}$ & $\mathrm{kN} / \mathrm{m}^{2}$ & Log-normal & 8,90 & 17,22 & Lindquist (2006) e Fonte (2004) \\
$\theta_{E}$ & - & Log-normal & 1,00 & 0,10 & JCSS (JOINT..., 2001) \\
$\theta_{R}$ & - & Normal & 1,00 & 0,10 & JCSS (JOINT..., 2001) \\
$L$ & $\mathrm{~m}$ & Determinística & 5,96 & - & Fonte (2004) \\
$B$ & $\mathrm{~m}$ & Determinística & 4,05 & - & Fonte (2004) \\
$e$ & $\mathrm{~m}$ & Determinística & 0,075 & - & Fonte (2004) \\
\hline
\end{tabular}

Nota: ${ }_{x}$ - média amostral; e $\sigma_{x}$ - desvio padrão amostral.

\section{Avaliação da confiabilidade estrutural}

A análise de confiabilidade foi realizada por meio da simulação de Monte Carlo para avaliar a probabilidade de falha independentemente do tempo, a qual foi também medida em termos do índice de confiabilidade $(\beta)$. Para isso foi implementado um programa computacional, conforme pode ser visualizado na Figura 9. Mais detalhes da simulação de Monte Carlo podem ser encontrados no trabalho de Cheung, Pinto e Calil Junior (2012). Para representar as variáveis aleatórias não gaussianas foi utilizada a transformação de Nataf, conforme recomendado por Hasofer e Lind (1974) e Hasofer (1974). Para o cálculo da probabilidade de falha cada caminhão foi simulado isoladamente e aplicado o teorema da probabilidade total, conforme a Equação 10, para a obtenção da probabilidade de falha do sistema $\left(P_{f, \text { sistema }}\right)$, sendo $N C$ o número de caminhões, $C_{i}$ os tipos de caminhões (carregamento) avaliados e $G$ a relação de estado limite.

$P_{f, \text { sistema }}=\sum_{i=1}^{N C} P\left[G<0 \mid C_{i}\right] \cdot P\left[C_{i}\right]$

Eq. 10

Os índices de confiabilidade obtidos com as simulações que envolveram cada um dos 12 tipos de veículos avaliados, assim como do sistema, foram comparados com os valores sugeridos pelo Joint Committee on Structural Safety (JOINT..., 2001), e com o valor estipulado de $\beta_{t}=4,7$.

\section{Resultados e discussão}

A Figura 10 apresenta os resultados da análise de confiabilidade da ponte para caminhões diferentes, considerando a carga estática com fator dinâmico.

Os resultados apresentam diferença entre o índice de confiabilidade alvo $\left(\beta_{t}=4,7\right)$ e os obtidos com caminhões $2 \mathrm{~S} 3\left(\beta_{2 S 3}=4,68\right)$.

$\mathrm{O}$ índice de confiabilidade do sistema $\left(\beta_{\text {sistema }}=4,64\right)$ demonstrou um valor abaixo dos valores recomendados pelo JCSS (JOINT..., 2001). Por outro lado, é importante lembrar que alguns caminhões com sobrecargas ilegais têm sido observados. O número de multas por violação de peso tem aumentado, conforme Harry et al. (2003).

Os resultados apresentados na Figura 9 indicam que a ponte do Rio Monjolinho avaliada atende aos requisitos de segurança propostos pelo JCSS (JOINT..., 2001), apesar de apresentar uma pequena diferença para o caminhão 2S3. Contudo, é necessária a análise de um modelo teórico para avaliar as perdas de protensão que seja mais representativo. É importante lembrar que a probabilidade de falha depende do modelo estrutural e que neste trabalho utilizou-se um modelo simplificado para representar esse problema, que é geralmente utilizado nas pontes protendidas de madeira.

\section{Conclusões}

Foi demonstrado que o caminhão $2 S 3$ é o que apresenta o menor índice de confiabilidade para a ponte de madeira sobre o Rio Monjolinho. No entanto, o sistema estrutural apresenta um bom índice de confiabilidade $(\beta)$ quando comparado com o índice de confiabilidade-alvo estipulado pelo JCSS (JOINT..., 2001). 
Figura 9 - Tela principal do programa computacional para a obtenção da probabilidade de falha e do índice de confiabilidade utilizando a simulação de Monte Carlo

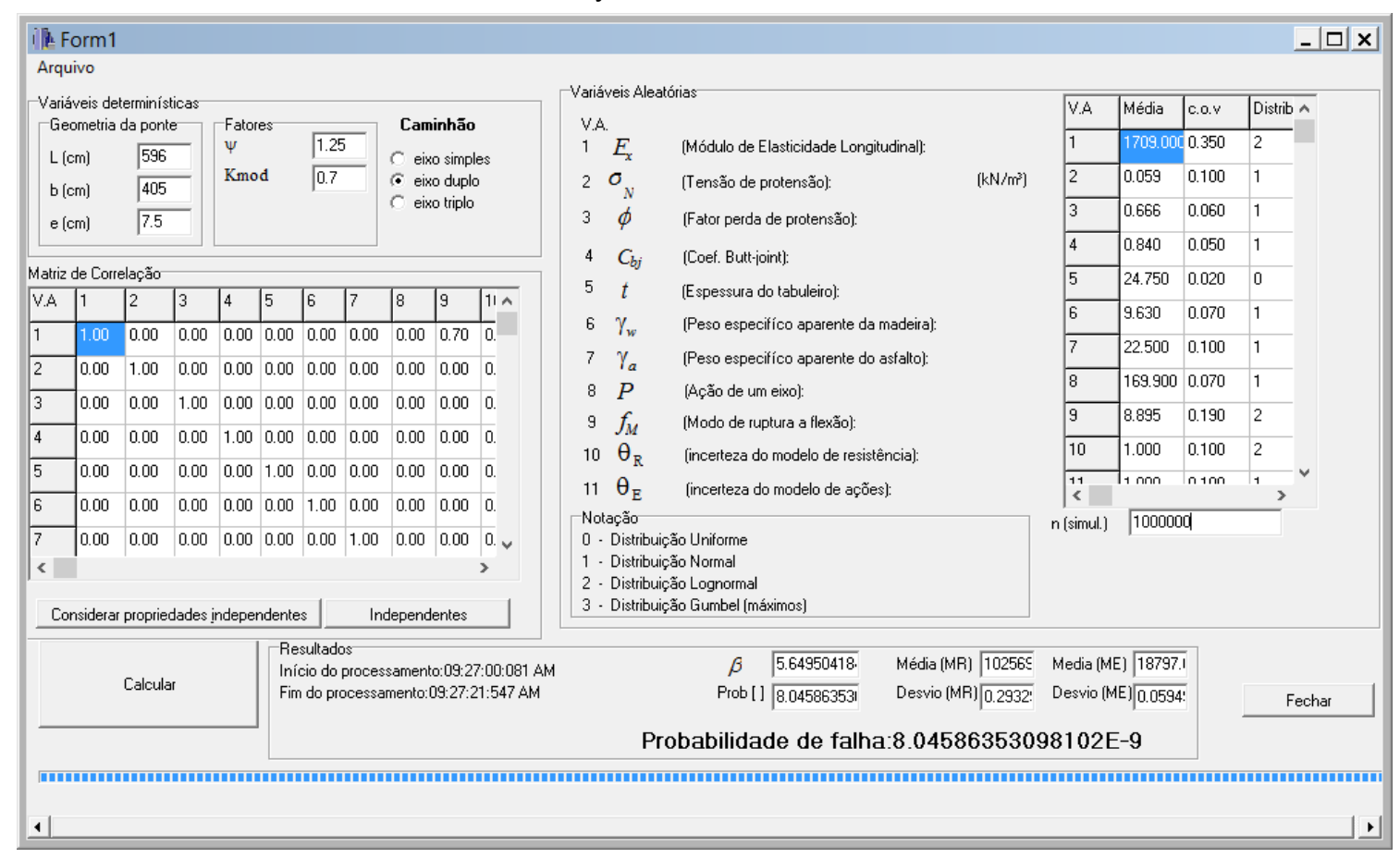

Figura 10 - Índice de confiabilidade para os caminhões avaliados

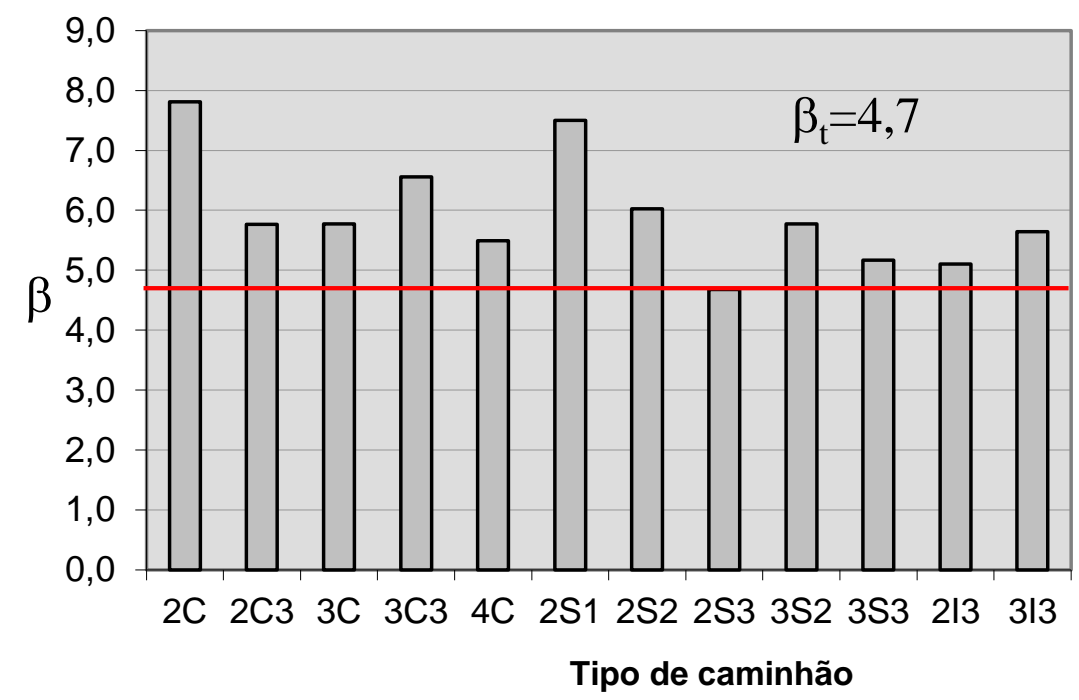

A perda de protensão foi considerada como variável aleatória no tempo inicial, o que consiste em um modelo simplificado. Dessa forma, é necessário avaliar a confiabilidade dependente do tempo para considerar corretamente as perdas de protensão com um modelo que incorpore um comportamento viscoelástico do material. Diferenças mais significativas podem ser esperadas quando modelos teóricos mais refinados forem aplicados para as perdas de protensão, o que dependerá de trabalhos experimentais sobre o assunto.

A análise apresentada da ponte confirma os resultados do estudo de Lindquist (2006), em que a confiabilidade do tabuleiro protendido, concebido de acordo com o procedimento da largura efetiva (RITTER, 1990), está de acordo com JCSS (JOINT..., 2001) em termos de segurança.

O método aqui apresentado pode também ser usado para estimar os possíveis impactos nas 
pontes de madeira, como resultado de mudanças dos carregamentos dos caminhões em função do avanço tecnológico da indústria automobilística e sobrecargas ilegais em rodovias. Pode ser aplicado também no planejamento de reprotensão e inspeção de pontes de madeira com base no método de confiabilidade invariante no tempo.

\section{Referências}

ASSOCIAÇÃO BRASILEIRA DE NORMA TÉCNICAS. NBR 7190: projeto de estruturas de madeira. Rio de Janeiro, 1997.

CALIL JUNiOR, C. Manual de Projeto e Construção de Pontes de Madeira. São Carlos: Fundação de Amparo a Pesquisa do Estado de São Paulo, 2006.

CHEUNG, A. B.; PINTO, E. M.; CALIL JUNIOR, C. Confiabilidade Estrutural de Vigas de Madeiras Submetidas à Flexão em Condições Normais e em Situação de Incêndio. Madeira: Arquitetura e Engenharia, v. 12, p. 1-12, 2012.

CHEUNG, A. B.; CALIL JUNIOR, C. Tabuleiro Ortótropo Treliçado Protendido Transversalmente Para Aplicação em Pontes de Madeira. Cadernos de Engenharia de Estruturas, v. 7, p. 79-110, 2006.

CHEUNG, A. B.; LINDQUIST, M.; CALIL JUNIOR, C. Calibração das Propriedades Elásticas de Uma Placa Ortótropa Utilizando Algoritmos Genéticos. Revista Sul-Americana de Engenharia Estrutural, v. 1, n. 2, p. 75-95, 2004.

CHEUNG, A. B. Tabuleiro Ortótropo Treliçado Protendido Transversalmente Para Aplicação em Pontes de Madeira. São Paulo, 2003. Dissertação (Mestrado em Engenharia de Estruturas) - Escola de Engenharia, Universidade de São Paulo, São Carlos, 2003.

DAHL, K.; BOVIM, N. I.; MALO, K. A. Evaluation of Stress Laminated Bridge Decks Based on Full Scale Tests. In: WORLD CONFERENCE ON TIMBER ENGINEERING, 10., Oregon, 2006. Proceedings... Oregon, 2006.

EAMON, C. et al. Reliability-Based Criteria for Load and Resistance Factor Design Code for Wood Bridges. Journal of the Transportation Research Board, 2000.

FONTE, T. F. Pontes protendidas de Eucaliptus citriodora. São Carlos, 2004. Dissertação (Mestrado em Engenhara de Estruturas) - Escola de Engenharia de São Carlos, Universidade de São Paulo, São Carlos, 2004.
GÓES, J. L. N.; DIAS, A. A. Comparação de Três Métodos de Cálculo Para Pontes de Madeira Protendida Transversalmente. Revista SulAmericana de Engenharia Estrutural, Passo Fundo, v. 2, p. 79-94, 2005.

GÓES, J. L. N. et al. Programa Para Análise de Placas Ortotrópicas. Revista Minerva, v. 3, p. 921, 2006.

HARRY, C. et al. Predicting Truck Load Spectra Under Weight Limit Changes and Its Application to Steel Bridge Fatigue Assessment. Journal of Bridge Engineering, v. 8, n. 5, p. 312-22, 2003.

HASOFER, A. M.; LIND, N. An Exact and Invariant First-Order Reliability Format. Journal of Engineering Mechanics, v. 100, n. EM1, p. 111-121, 1974.

HASOFER, A. M. Reliability Index and Failure Probability. Journal of Structural Mechanics, v. 3, p. 25-27, 1974.

\section{JOINT COMMITTEE ON STRUCTURAL} SAFETY. Probabilistic Model Code. 2001.

LINDQUIST, M. Confiabilidade Estrutural de Pontes Laminadas Protendidas de Madeira. São Carlos, 2006. Tese (Doutorado em Engenharia de Estruturas) - Escola de Engenharia de São Carlos, Universidade de São Paulo, São Carlos, 2006.

LINDQUIST, M. et al. Structural Reliability of a Stress Laminated Timber Bridge. In: INTERNATIONAL CONFERENCE ON PROBABILISTIC MODELS IN TIMBER ENGINEERING, Arcachon, 2005. Proceedings... Arcachon: Association Arbora, 2005.

OKIMOTO, F. S. Pontes Protendidas de Madeira: parâmetros de projeto. São Carlos, 1997. Dissertação (Mestrado em Engenharia de Estruturas) - Escola de Engenharia de São Carlos, Universidade de São Paulo, São Carlos, 1997.

OLIVA, M. G.; DIMAKIS, A. Behaviour of Stress-Laminated Timber Highway Bridges. Journal of Structural Engineering, v. 114, n. 8, p. 1850-1869, 1988.

RITTER, M. A. Timber Bridges: design, construction, inspection, and maintenance. Washington: United States Department of Agriculture Forest Service, 1990.

TAYLOR, R. J.; BATCHELOR, B. D.; VAN DALEN, K. Prestressed Wood Bridges. Toronto: Canadian Soc. for Civil Engineering, 1982. 


\section{Andrés Batista Cheung}

Departamento de Engenharia Civil | Universidade Federal de Mato Grosso do Sul | Cidade Universitária, Centro | Caixa Postal 549 | Campo Grande - MS - Brasil | CEP 79070-900 | Tel.: (67) 3345-7376 Ramal 7376 | E-mail: andres.cheung@ufms.br

\section{Ricardo de Mello Scaliante}

Superintendência Regional | Departamento Nacional de Infraestrutura de Transportes de Mato Graosso do Sul | Rua Antônio Maria Coelho, 3099, Centro | Campo Grande - MS - Brasil | CEP 79020-916 | Tel.: (67) 3302-5772 | E-mail: ricscaliante@yahoo.com.br

\section{Malton Lindquist}

Secretaria da Fazenda do Estado do Ceará | Rua Pessoa Anta, 274, Centro | Fortaleza - CE - Brasil | CEP 60060-430 | Tel.: (88) 3102-1113 | E-mail: malton@sc.usp.br

\section{André Luis Christoforo}

Departamento de Engenharia Civil | Universidade Federal de São Carlos | Rodovia Washington Luís, km 235 - SP-310 | São Carlos - SP -

Brasil | CEP 36307-352 | Tel.: (16) 3351-8262 | E-mail: alchristoforo@gmail.com

\section{Carlito Calil Junior}

Departamento de Estruturas, Escola de Engenharia de São Carlos | Universidade de São Paulo | Av. Trabalhador Saocarlense, 400, Centro | Caixa Postal 359 | São Carlos - SP - Brasil | CEP 13566-590 | Tel.: (16) 3373-9320 | E-mail: calil@sc.usp.br

\section{Revista Ambiente Construído}

Associação Nacional de Tecnologia do Ambiente Construído

Av. Osvaldo Aranha, $99-3^{\circ}$ andar, Centro

Porto Alegre - RS - Brasil

CEP $90035-190$

Telefone: +55 (51) 3308-4084

Fax: +55 (51) 3308-4054

www.seer.ufrgs.br/ambienteconstruido

E-mail: ambienteconstruido@ufrgs.br 